\title{
Mid-infrared laser-based detection of benzene
}

Shakfa, Mohammad Khaled, Elkhazraji, Ali, Marangoni, Marco, Farooq, Aamir

Mohammad Khaled Shakfa, Ali Elkhazraji, Marco Marangoni, Aamir Farooq, "Mid-infrared laser-based detection of benzene," Proc. SPIE 11772, Optical Sensors 2021, 1177217 (18 April 2021); doi: 10.1117/12.2595041

SPIE. Event: SPIE Optics + Optoelectronics, 2021, Online Only 


\title{
Mid-infrared laser-based detection of benzene
}

\author{
Mohammad Khaled Shakfa ${ }^{\mathrm{a}, 1}$, Ali Elkhazraji ${ }^{\mathrm{a}}$, Marco Marangoni ${ }^{\mathrm{b}}$, and Aamir Farooq ${ }^{\mathrm{a}}$ \\ ${ }^{a}$ King Abdullah University of Science and Technology (KAUST), Physical Science and Engineering \\ Division, Clean Combustion Research Center (CCRC), Thuwal 23955-6900, Saudi Arabia \\ ${ }^{b}$ Politecnico di Milano, Department of Physics and IFN-CNR, Via G. Previati 1/C, 23900 Lecco, \\ Italy
}

\begin{abstract}
Benzene $\left(\mathrm{C}_{6} \mathrm{H}_{6}\right)$ is one of the major public health concerns. It is emitted from various natural and anthropogenic sources, like fires and volcanic emissions, petrol service stations, transportation, and the plastics industry. Here, we present our work on developing a new benzene sensor using a widely tunable difference-frequency-generation (DFG) laser emitting between 11.56 and $15 \mu \mathrm{m}\left(667-865 \mathrm{~cm}^{-1}\right)$. The DFG process was realized between an external-cavity quantum-cascadelaser and a $\mathrm{CO}_{2}$ gas laser in a nonlinear, orientation-patterned GaAs crystal. We obtained the absorption cross-sections of the Q-branch of the $v_{4}$ vibrational band of benzene by tuning the wavelength of the DFG laser between 14.79 and 14.93 $\mu \mathrm{m}\left(670-676 \mathrm{~cm}^{-1}\right)$. Benzene sensing measurements were performed near $14.84 \mu \mathrm{m}\left(673.97 \mathrm{~cm}^{-1}\right)$ with a direct laser absorption spectroscopy scheme. The benzene concentration was varied between ppb and ppm levels. Even with a relatively short optical path-length of $23 \mathrm{~cm}$, our sensor achieved a benzene detection limit of about $10 \mathrm{ppb}$.
\end{abstract}

Keywords: Difference frequency generation, mid-infrared laser sources, benzene, absorption cross-section, laser spectroscopy, laser sensor.

\section{INTRODUCTION}

Air pollution monitoring has attracted increasing attention and has become more and more important due to the significant impacts of pollution on public health as well as the global atmosphere. Global (greenhouse) and local emissions have significantly contributed to the increase in the level of air pollution, and it is of high importance to measure and control them. These emissions include volatile organic compounds (VOCs), with benzene as one of its main compounds of high interest. Benzene $\left(\mathrm{C}_{6} \mathrm{H}_{6}\right)$ is one of the major public health concerns, and it is emitted from various human-made and natural sources, like volcanic emissions, burning of fossil fuels, transportation, solvent use, and industrial processes. Benzene is classified as a hazardous and carcinogenic air pollutant by the International Agency for Research on Cancer (IARC) and the World Health Organization (WHO) $)^{1,2}$. Short-term exposure to a concentration as low as $25 \mathrm{ppm}$ can cause insomnia, paresthesia, nausea, and speech problems. Exposure to even higher concentrations, e.g., 20,000 ppm, for 5-10 minutes may result in death due to central nervous system depression, asphyxia, or respiratory arrest ${ }^{3,4}$. The most threatening effects on the health of long-term exposure to benzene are genotoxicity (DNA toxicity), haematotoxicity (blood cells toxicity), and carcinogenicity ${ }^{1}$. However, according to WHO, there is no minimum level of benzene exposure that could be safely recommended due to the severe health risks it can bring about ${ }^{1}$. Besides the health risk factors, innovating new sensors and detection schemes for benzene is very important in a number of applications such as space research ${ }^{5,6}$ and combustion diagnostics ${ }^{7,8}$.

Laser-based sensors, particularly in the mid-infrared (MIR) region, enable non-intrusive, quantitative, fast response time measurements of temperature, pressure, and composition of chemical species with high spatial and temporal resolution. Such sensors have been shown great potential for benzene detection. Laser-based benzene sensors have focused on the MIR absorption band near $3.3 \mu \mathrm{m}^{9}$ and $3.4 \mu \mathrm{m}^{10}$. However, the detection near these wavelengths suffers from considerable spectral overlap with other VOCs, ethylene, and methane. Benzene sensors were also developed near $9.64 \mu \mathrm{m}$ with spectral interferences mainly from ozone and ethylene ${ }^{11}$. Besides, Sydoryk et al. reported benzene detection between $9.41 \mu \mathrm{m}$ and $9.88 \mu \mathrm{m}^{12}$. Benzene was also detected near $14.8 \mu \mathrm{m}$ using a difference-frequency-generation (DFG) laser source with a relatively high signal-to-noise ratio ${ }^{13}$. Recently, benzene detection near $14.84 \mu \mathrm{m}$ has been reported employing cantileverenhanced photoacoustic spectroscopy (CEPAS) with a long-wavelength quantum cascade laser operating at $-23{ }^{\circ} \mathrm{C}^{14}$.

\footnotetext{
${ }^{1}$ khaled.shakfa@kaust.edu.sa
} 
However, the CEPAS cell had to be heated to $50{ }^{\circ} \mathrm{C}$ with a mixture pressure of 200 mbar. The only relevant interference near $14.84 \mu \mathrm{m}$ is $\mathrm{CO}_{2}$.

Here, we present our work on benzene detection near $14.84 \mu \mathrm{m}$ using a widely tunable laser emitting between $11.56 \mu \mathrm{m}$ and $15 \mu \mathrm{m}$. Thanks to the unique feature of fine tunability of our laser, we obtained the absorption cross-sections of the Qbranch of the $v_{4}$ vibrational band of benzene with a relatively low spectral spacing. The benzene concentration was varied between ppb and ppm levels and measured in a direct laser absorption spectroscopy scheme with a gas cell of a $23 \mathrm{~cm}$ length.

\section{EXPERIMENTAL SETUP}

Figure 1 shows an experimental layout of the DFG laser source together with the setup for gas sensing measurements. The laser source is based on the DFG interaction between the radiation emitted from an external-cavity quantum-cascade-laser (EC-QCL) (pump source) and a CO2 gas laser (signal source). The EC-QCL is mode-hop-free tunable between 5.45 and $5.71 \mu \mathrm{m}\left(1749.97-1834.93 \mathrm{~cm}^{-1}\right)$, and the $\mathrm{CO}_{2}$ gas laser is non-continuously tunable between 9.23 and $10.86 \mu \mathrm{m}(920.81-$ $1083.42 \mathrm{~cm}^{-1}$ ). Both pump and signal lasers were collinearly combined using a beam combiner and focused onto a nonlinear orientation-patterned gallium arsenide (OP-GaAs) crystal. Thereby, the idler laser can be tuned over a broad range between 11.56 and $15 \mu \mathrm{m}\left(666.53-865.28 \mathrm{~cm}^{-1}\right)$ with a laser linewidth of $\sim 2.3 \mathrm{MHz}$ and output power of up to 31 $\mu \mathrm{W}$. A long-pass filter (LPF), with a cut-on edge near $11.2 \mu \mathrm{m}$, was used to filter out both the pump and signal lasers. More details on our DFG laser can be found elsewhere ${ }^{15}$. The DFG laser setup was placed in a sealed box purged with nitrogen to reduce the effect of ambient water absorption, i.e., strong water absorption lines existing in the EC-QCL spectrum, on the measurements. The $\mathrm{CO}_{2}$ gas laser was operated in pulse mode with a pulse repetition rate of $2.5 \mathrm{kHz}$ and a duty cycle of $25 \%$.

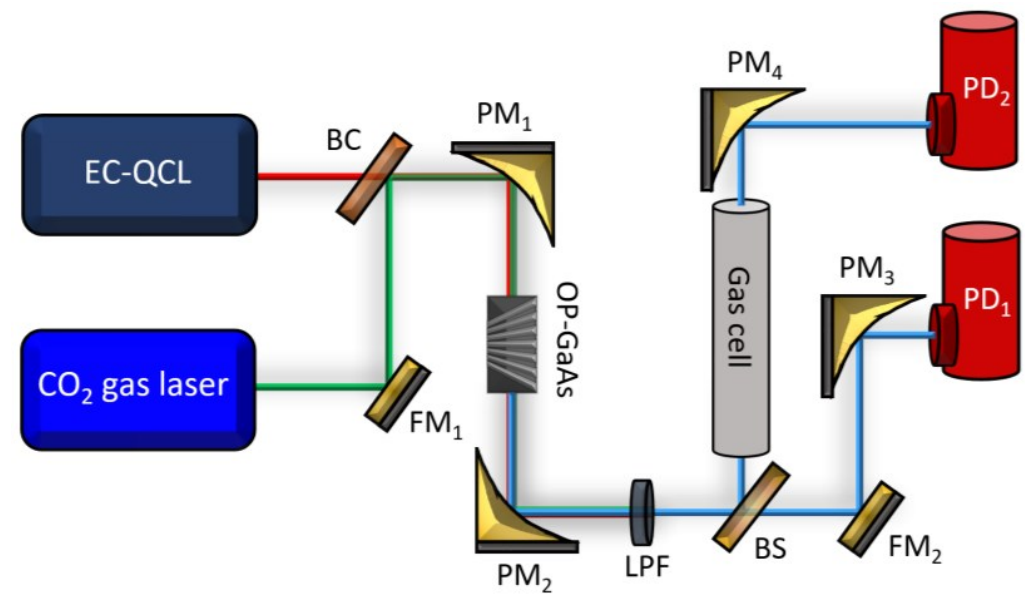

Figure 1. Experimental layout of the DFG laser source together with the setup for gas sensing measurements. EC-QCL: external-cavity quantum cascade laser, FM: flat mirror; BC: beam combiner; PM: parabolic mirror; OP-GaAs: orientationpatterned GaAs crystal; LPF: long-pass filter; BS: beam splitter; PD: photodetector.

After the LPF, a beam splitter was installed to split the idler laser beam into two beams: (i) the transmitted beam was directly focused onto a liquid-nitrogen-cooled $\mathrm{HgCdTe}$ photodetector $\left(\mathrm{PD}_{1}\right)$ using a parabolic mirror $(\mathrm{PM})$, and (ii) the reflected beam was aligned through a 23-cm-length optical cell (gas cell) via coated-ZnSe windows and then focused onto another liquid-nitrogen-cooled $\mathrm{HgCdTe}$ detector $\left(\mathrm{PD}_{2}\right)$. The signal on $\mathrm{PD}_{1}$ was used as a reference, while the signal on $\mathrm{PD}_{2}$ was used for sensing measurements. We used a digital oscilloscope to record detectors' signals which were postprocessed and analyzed using MATLAB and OriginLab software. For sensing measurements, benzene concentration was diluted using nitrogen gas. We prepared several benzene/nitrogen mixtures with benzene concentrations varying from ppblevel to ppm-level.

\section{MEASUREMENT PRINCIPLE AND UNCERTAINTY}

We applied the Beer-Lambert law to obtain the absorption cross-section and benzene concentration. When a laser beam is passed through an absorber-medium, e.g., gas mixture, the attenuation of this beam, due to absorption, can be related to the physical parameters of the gas and the experimental conditions via 


$$
-\ln \left(\frac{I_{t}(v)}{I_{0}(v)}\right) \equiv A(v, T, P)=\sigma(v, T, P) n L
$$

where $\mathrm{I}_{\mathrm{t}}$ and $\mathrm{I}_{0}$ are the transmitted and incident laser intensities, respectively, at a frequency $(v), A$ is the absorbance, $\sigma$ is the absorption cross-section, $\mathrm{T}$ is the temperature, $\mathrm{P}$ is the pressure, $\mathrm{n}$ is the number density of the absorbing species, and $\mathrm{L}$ is the absorption path length. The relation between the mole fraction $(\mathrm{x})$ of the absorbing species and the number density is:

$$
n=\frac{N}{V}=\frac{x P}{k_{B} T}
$$

where $\mathrm{N}$ is the number of absorbers in the volume $\mathrm{V}$ and $\mathrm{k}_{\mathrm{B}}$ is the Boltzmann constant $\left(\mathrm{k}_{\mathrm{B}}=1.38 \times 10^{-23} \mathrm{~J} / \mathrm{K}\right)$. From Eq. (1) and (2), one can get the relation between the absorption cross-section and the experimental parameters as:

$$
\sigma(v, T, P)=\frac{A(v, T, P) k_{B} T}{x p L}
$$

One can use Eq. (3) to calculate the absorption cross-section (or the mole fraction) when other parameters are known. The uncertainty in the absorption cross-section is obtained as follows,

$$
\frac{\delta \sigma}{\sigma}=\sqrt{\left(\frac{\delta A}{A}\right)^{2}+\left(\frac{\delta T}{T}\right)^{2}+\left(\frac{\delta x}{x}\right)^{2}+\left(\frac{\delta P}{P}\right)^{2}}
$$

while the uncertainty in the mole fraction is obtained from

$$
\frac{\delta x}{x}=\sqrt{\left(\frac{\delta A}{A}\right)^{2}+\left(\frac{\delta T}{T}\right)^{2}+\left(\frac{\delta \sigma}{\sigma}\right)^{2}+\left(\frac{\delta P}{P}\right)^{2}}
$$

For measurements at a fixed wavelength, the uncertainty in the absorbance is obtained from

$$
\frac{\delta A}{A}=\frac{\delta\left(\frac{I_{t}}{I_{0}}\right)}{\left(\frac{I_{t}}{I_{0}}\right)}
$$

where the uncertainty in the intensity ratio $I_{t} / I_{0}$ is the standard deviation of the mean value of the ratio. In contrast, for scan measurements, $\delta \mathrm{A}$ is determined from the fluctuation of the absorbance values in a relatively flat spectral region or from the noise fluctuation at a zero absorption area. All measurements presented in this paper were measured at room temperature of $\sim 295 \mathrm{~K}$ and atmospheric pressure. The uncertainty in the temperature is $0.1 \mathrm{~K}$, and the uncertainty in the pressure is $0.12 \%$ of the pressure reading, i.e., $0.0012 \mathrm{~atm}$. The uncertainty in the mixture mole fraction is $2 \%$.

\section{WAVELENGTH SELECTION}

The PNNL (Pacific Northwest National Laboratory) database ${ }^{16}$ offers a broad IR absorption spectrum of benzene measured at $298 \mathrm{~K}$ and $1 \mathrm{~atm}$. We used Eq. (3) to calculate the absorption cross-section of benzene using the absorbance values from the PNNL database; the absorption cross-section of benzene is shown in Fig. 2(a). The strongest IR active band is the $v_{4}$ band (centered near $674 \mathrm{~cm}^{-1}$ ), which is related to the $\mathrm{C}-\mathrm{H}$ bending motion of benzene. It is worth mentioning that this band is sometimes called $v_{11}$ in the literature. This is due to different numbering conventions ${ }^{17}$. Despite the broad spectrum of the PNNL database, it is, however, at a low sampling rate, i.e., the spectral spacing is about $0.06 \mathrm{~cm}^{-1}$. The green circles in Fig. 2(b) are the PNNL absorption cross-sections of the Q branch of benzene. Benefiting from the fine scanning capability of our DFG laser, we performed some measurements to fully resolve the absorption spectrum of the Q branch of benzene. For these measurements, the $\mathrm{CO}_{2}$ gas laser wavelength was set to $9.24 \mu \mathrm{m}\left(1082.25 \mathrm{~cm}^{-1}\right)$, while the EC-QCL laser was swept between $5.6875 \mu \mathrm{m}\left(1758.25 \mathrm{~cm}^{-1}\right)$ and $5.7069 \mu \mathrm{m}\left(1752.25 \mathrm{~cm}^{-1}\right)$. The sweep measurements were repeated four times and overlayed to achieve a spectral spacing of $\sim 8 \times 10^{-4} \mathrm{~cm}^{-1}$. The blue line in Fig. 2(b) shows the absorption cross-sections of benzene obtained from our scan measurements of a benzene/nitrogen mixture with a benzene concentration of $260 \mathrm{ppm}$. To the best of our knowledge, this is the first, scanned laser-based absorption measurements of 
the whole Q branch of benzene. Our measurements reveal the maximum of the strongest absorption feature of benzene IR spectrum which was not clear in the PNNL database due to its relatively large spectral spacing.
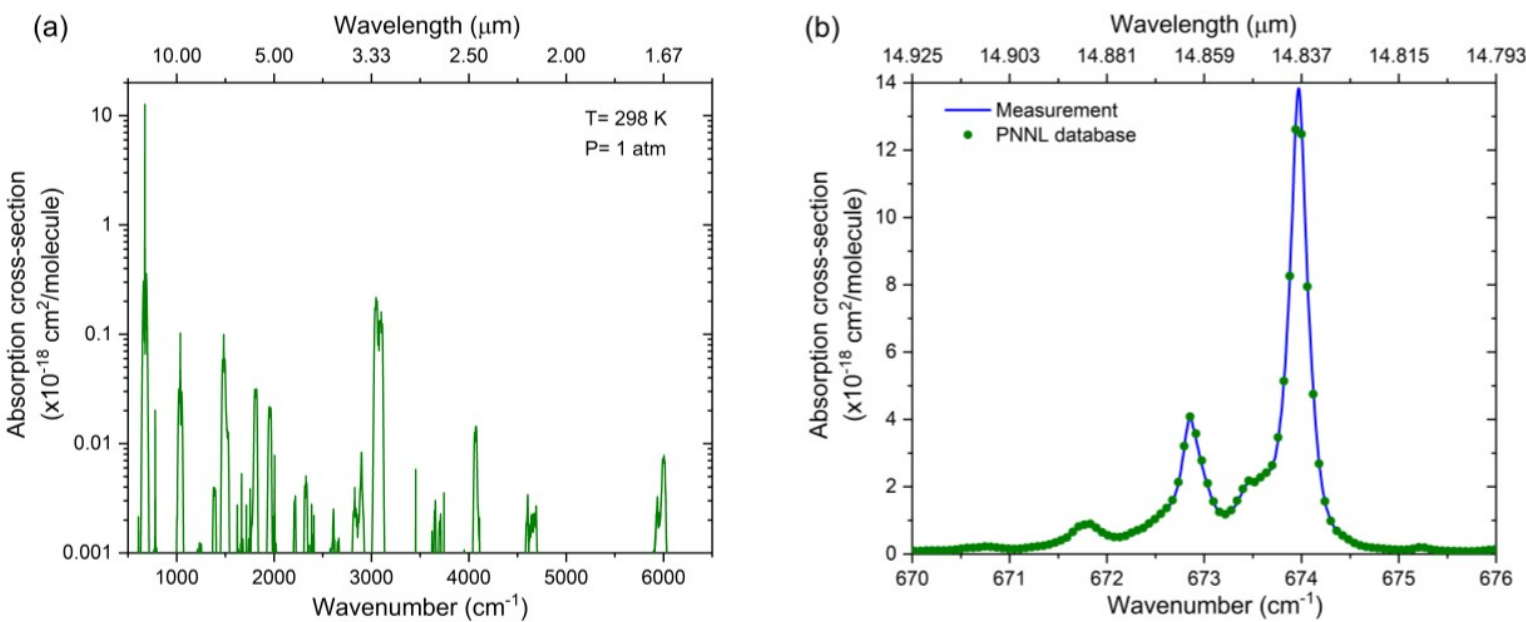

Figure 2. (a) Absorption cross-section of benzene in the IR region calculated from Eq. (3) using the absorbance values of the PNNL database. (b) Absorption cross-section of the Q branch of the $v_{4}$ vibrational band of benzene; green circles are obtained from the PNNL database, and the blue line is obtained from our scan measurements.
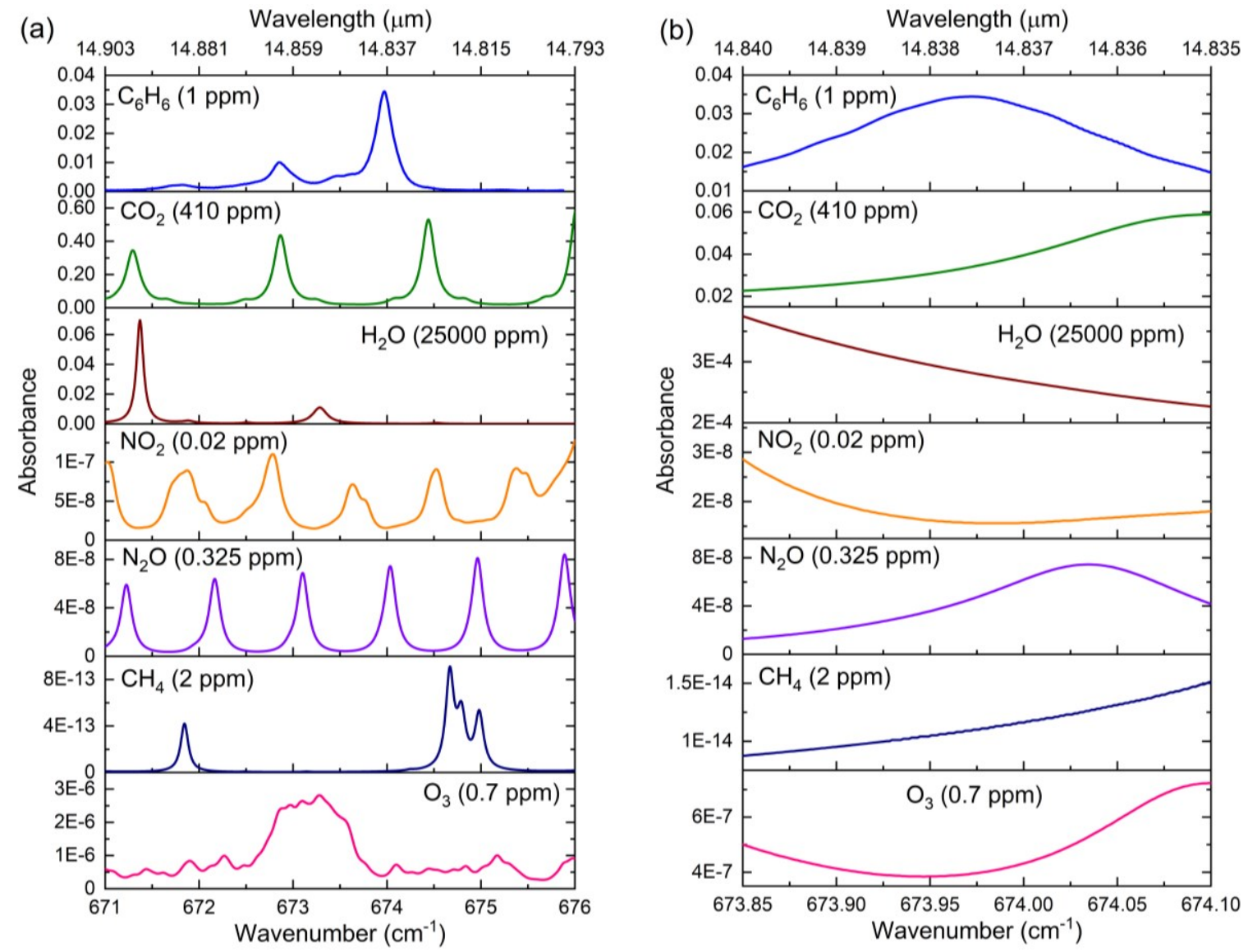

Figure 3. (a) Absorbance spectra of benzene, carbon dioxide, water, nitrogen dioxide, nitrous oxide, methane, and ozone for an optical path length of $1 \mathrm{~m}$ at room temperature and atmospheric pressure. (b) A magnification of the spectra of (a) around $673.97 \mathrm{~cm}^{-1}$. Except for benzene, all spectra are taken from the HITRAN database ${ }^{18}$. 
To determine the optimum wavelength for benzene sensing in ambient air applications, we plotted the absorbance spectrum of benzene with the spectra of air-contained species at their average-estimated concentrations. Figure 3(a) shows the Q branch spectrum of benzene at $1 \mathrm{ppm}$ together with the spectra of carbon dioxide, water, nitrogen dioxide, nitrous oxide, methane, and ozone. The benzene absorbance values are obtained based on the absorption cross-section of Fig. 2(b). There is no interference between the benzene spectrum and the absorption spectrum of carbon monoxide in the considered spectral range. One can see that the main interferences are with the spectra of carbon dioxide and water. For further clarification, we magnified the spectra around the maximum of the absorption feature of the Q branch of benzene, as shown in Fig. 3(b). Close to the center of the absorption feature, at $673.97 \mathrm{~cm}^{-1}(14.8374 \mu \mathrm{m})$, the absorbance value of benzene is higher by about two orders of magnitude than the value of the water absorbance, and it is almost equal to the absorbance value of carbon dioxide. Consequently, we set the DFG wavelength to $14.8374 \mu \mathrm{m}$ resulting from tuning the $\mathrm{CO}_{2}$ gas laser to $9.24 \mu \mathrm{m}$ and the EC-QCL to $5.694 \mu \mathrm{m}$ and performed sensing measurements at different benzene concentrations.

\section{SENSING MEASUREMENTS}

The $\mathrm{CO}_{2}$ gas laser (signal source), and hence the DFG laser (idler), was operated in pulsed mode with a pulse repetition rate of $2.5 \mathrm{kHz}$. We used the peak-to-peak (P-P) amplitude of the DFG laser pulses to determine the laser intensity. As shown in Fig. 1, the dual-beam configuration of the sensing setup allows us to eliminate any effect of laser intensity fluctuations on the measurements. We first obtained the ratio between the laser intensities on photodetectors when the gas cell was vacuumed. Then, we used this intensity ratio to normalize the laser signal of both detectors to obtain absorbance values when the gas cell is filled with benzene/nitrogen mixtures.

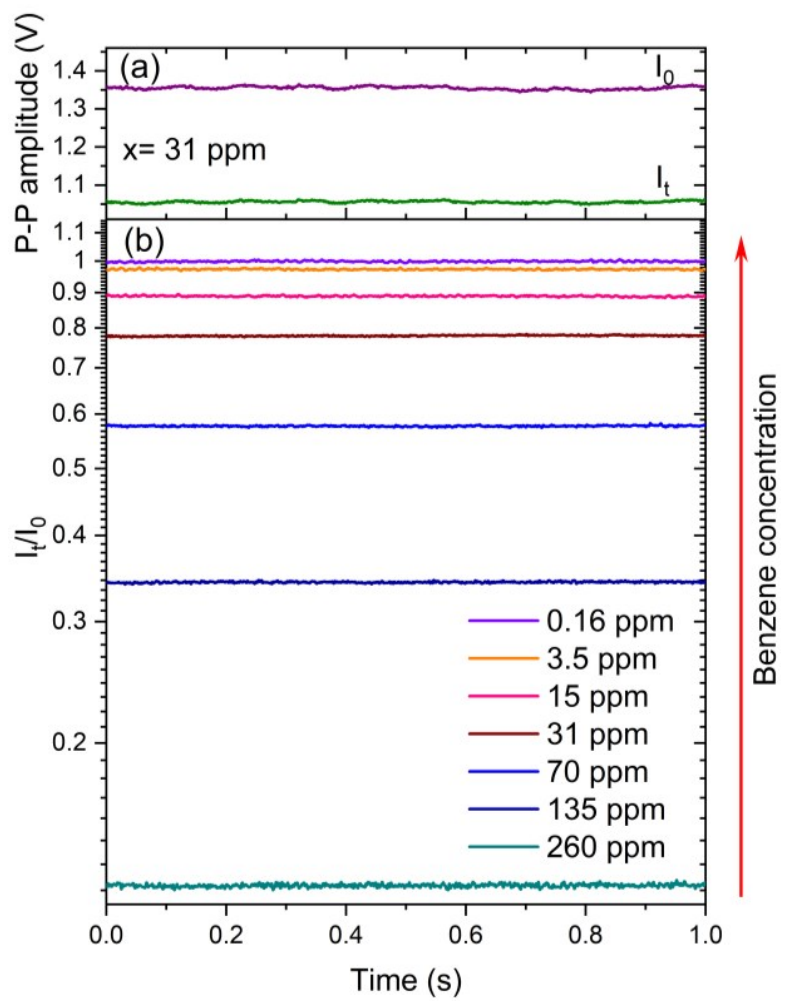

Figure 4. (a) Peak-to-peak (P-P) amplitudes of the transmitted ( $\left.\mathrm{I}_{t}\right)$ and incident $\left(\mathrm{I}_{0}\right)$ laser intensities for the mixture of a benzene concentration of $31 \mathrm{ppm}$. (b) The intensity ratio $\mathrm{I}_{\mathrm{t}} / \mathrm{I}_{0}$ of several benzene mixtures.

We prepared a number of benzene/nitrogen mixtures with known benzene concentrations varying between ppb and ppm levels. Figure 4 (a) shows an example of the P-P amplitudes of signals of $\mathrm{PD}_{1}$ and $\mathrm{PD}_{2}$, which represent the transmitted $\left(\mathrm{I}_{t}\right)$ and incident $\left(\mathrm{I}_{0}\right)$ laser intensities, respectively, for the mixture of a benzene concentration of $31 \mathrm{ppm}$. The apparent fluctuation in both, $\mathrm{I}_{\mathrm{t}}$ and $\mathrm{I}_{0}$ reflects the fluctuation in the $\mathrm{CO}_{2}$ gas laser intensity. However, thanks to the measurement principle, this fluctuation vanishes when calculating the intensity ratio $\mathrm{I}_{t} / \mathrm{I}_{0}$ to obtain the absorbance value and the benzene concentration using Eq. (3). Figure 4(b) shows the ratio $I_{t} / I_{0}$ of several benzene mixtures measured over a time period of one second. 
We applied Eq. (3) to obtain the benzene concentration in various benzene/nitrogen mixtures. The absorption cross-section was taken from Fig. 2(b) and is equal to $(13.83 \pm 0.28) \times 10^{-18} \mathrm{~cm}^{2} /$ molecule at $14.8374 \mu \mathrm{m}$. The considerable uncertainty of about $2 \%$ in the cross-section value is because it was obtained from a single mixture. However, this uncertainty can be reduced when the cross-section curve is obtained from different mixtures resulting in a decreased statistical error. Figure 5(a) shows the experimentally measured benzene concentration $\left(\mathrm{x}_{\mathrm{m}}\right)$ against the set (or known) concentration $\left(\mathrm{x}_{\mathrm{s}}\right)$. The red line represents the best $\mathrm{x}-\mathrm{y}$ axes correlation and is plotted to demonstrate the very good match between the measured and set values of the benzene concentration. The uncertainty in the measured benzene concentration was calculated using Eq. (5), and it was found to be $2 \%$ of the measured concentration for all mixtures. We also calculated the relative difference between the set and measured values of the benzene concentration, as shown in Fig. 5 (b). Except for benzene concentrations below $600 \mathrm{ppb}$, the relative difference is in the range of the uncertainty of the measured benzene concentrations. Below $600 \mathrm{ppb}$, the relative difference increases to about $4 \%$, which could be due to the multiple dilutionprocess used for preparing the mixtures to reach such low benzene concentrations.

In addition to the uncertainty in the measured absorption cross-section of benzene, the uncertainty in the measured benzene concentration reflects the fluctuation in the intensity ratio $\mathrm{I}_{t} / \mathrm{I}_{0}$ shown in Fig. 4(b). By taking the detection limit as three times the standard deviation, the detection limit in our study is $3 \times 3.26 \mathrm{ppb}$, where $3.26 \mathrm{ppb}$ is the uncertainty of the lowest detected benzene concentration, which was calculated using Eq. (5).

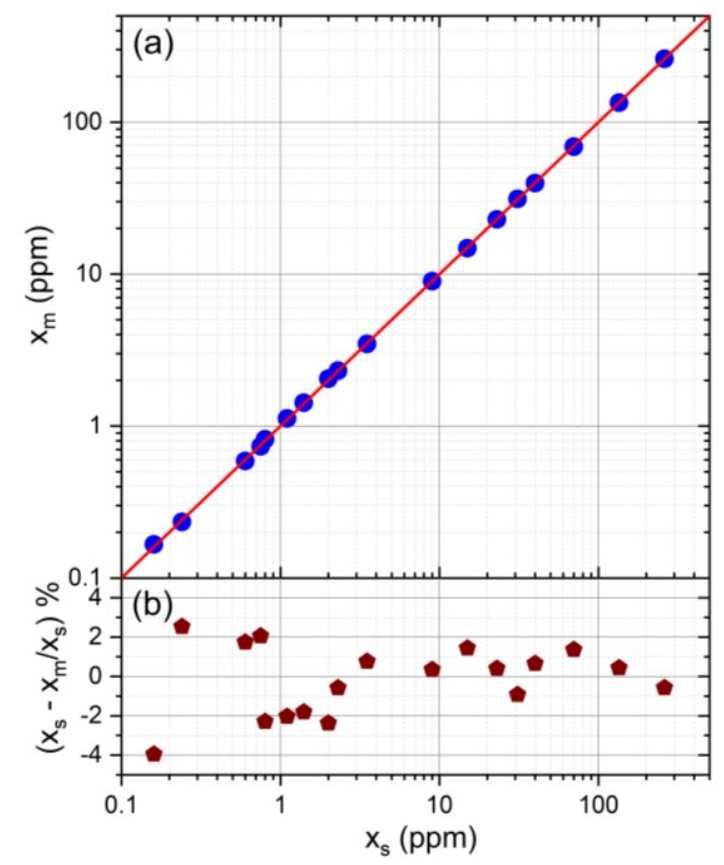

Figure 5. (a) Experimentally measured benzene concentration $\left(\mathrm{x}_{\mathrm{m}}\right)$ against the set concentration $\left(\mathrm{x}_{\mathrm{s}}\right)$. The red line represents the best correlation between $\mathrm{x}$ and $\mathrm{y}$ axes. (b) The relative difference between the set and measured benzene concentrations.

\section{SUMMARY AND OUTLOOK}

We have demonstrated a laser-based sensor for trace gas sensing of benzene. As a laser source for this sensor, we employed a widely tunable difference-frequency-generation (DFG) laser emitting between 11.56 and $15 \mu \mathrm{m}\left(667-865 \mathrm{~cm}^{-1}\right)$. We obtained the absorption cross-sections of the Q-branch of the strongest IR active band i.e., $v_{4}$ vibrational band, of benzene. We have identified the optimal spectral range for benzene sensing measurements, in which the absorption spectrum of benzene has minimum interferences with the spectra of air-contained species. For benzene sensing measurements, we tuned the DFG laser to $14.8374 \mu \mathrm{m}\left(673.97 \mathrm{~cm}^{-1}\right)$. At this wavelength, the primary absorption spectral overlap is from carbon dioxide. We applied our sensor to detect benzene in various benzene/nitrogen mixtures with benzene concentrations varying between ppb and ppm levels. A benzene concentration of $160 \mathrm{ppb}$ was detected with a detection limit of about 10 ppb. We believe that employing multi-pass cell or an enhanced cavity will significantly improve the detection limit down to ppt levels. Furthermore, thanks to the broad tunability of the used DFG laser, our sensor can be used for multi-species detection for air quality monitoring applications, in particular, to detect benzene and carbon dioxide simultaneously. 


\section{ACKNOWLEDGMENTS}

This work was funded by the Office of Sponsored Research (OSR) at King Abdullah University of Science and Technology (KAUST).

\section{REFERENCES}

[1] World Health Organization, [Air quality guidelines for Europe (2nd Edition)], WHO Regional Publications, European Series, No. 91, Copenhagen (2000).

[2] World Health Organization, [Benzene - IARC Monograph on the Evaluation of Carcinogenic Risks to Humans], International Agency for Research on Cancer, Lyon (2018).

[3] World Health Organization, [WHO Guidelines for Indoor Air Quality - Selected Pollutants], WHO Regional Office for Europe, Copenhagen (2010).

[4] Stücker, I., Mandereau, L., Aubert-Berleur, M. P., Déplan, F., Paris, A., Richard, A. and Hémon, D., "Occupational paternal exposure to benzene and risk of spontaneous abortion," Occup. Environ. Med. 51(7), 475478 (1994).

[5] Bézard, B., Drossart, P., Encrenaz, T. and Feuchtgruber, H., "Benzene on the giant planets," Icarus 154(2), 492$500(2001)$.

[6] Vinatier, S., Schmitt, B., Bézard, B., Rannou, P., Dauphin, C., de Kok, R., Jennings, D. E. and Flasar, F. M., "Study of Titan's fall southern stratospheric polar cloud composition with Cassini/CIRS: Detection of benzene ice," Icarus 310, 89-104 (2018).

[7] Böhm, H., Jander, H., and Tanke D., "PAH growth and soot formation in the pyrolysis of acetylene and benzene at high temperatures and pressures: Modeling and experiment", Proc. Combust. Inst. 27, 1605-1612 (1998).

[8] Shakfa, M. K., Mhanna, M., Jin, H., Liu, D., Djebbi, K., Marangoni, M. and Farooq, A., "A mid-infrared diagnostic for benzene using a tunable difference-frequency-generation laser," Proc. Combust. Inst. 38, 1787$1796(2021)$.

[9] Mhanna, M., Zhang, G., Kunnummal, N. and Farooq, A., "Cavity-Enhanced Measurements of Benzene for Environmental Monitoring," IEEE Sens. J. 21(3), 3849-3859 (2021).

[10] Sur, R., Ding, Y., Jackson, R. B. and Hanson, R. K., "Tunable laser-based detection of benzene using spectrally narrow absorption features," Appl. Phys. B Lasers Opt. 125(11), 1-8 (2019).

[11] Lewicki, R., Witinski, M., Li, B. and Wysocki, G., "Spectroscopic benzene detection using a broadband monolithic DFB-QCL array,” Proc. SPIE 9767, Novel In-Plane Semiconductor Lasers XV, 97671T (2016)

[12] Sydoryk, I., Lim, A., Jäger, W., Tulip, J. and Parsons, M. T., "Detection of benzene and toluene gases using a mid-infrared continuous-wave external cavity quantum cascade laser at atmospheric pressure," Appl. Opt. 49(6), 945-949 (2010).

[13] Chen, W., Cazier, F., Tittel, F. and Boucher, D., "Measurements of Benzene Concentration by DifferenceFrequency Laser Absorption Spectroscopy," Appl. Opt. 39(33), 6238-6242 (2000).

[14] Karhu, J., Philip, H., Baranov, A., Teissier, R. and Hieta, T., "Sub-ppb detection of benzene using cantileverenhanced photoacoustic spectroscopy with a long-wavelength infrared quantum cascade laser," Opt. Lett. 45(21), $5962(2020)$.

[15] Shakfa, M. K., Lamperti, M., Gotti, R., Gatti, D., Elkhazraji, A., Hakimov, K., Marangoni, M. and Farooq, A., "A widely tunable difference-frequency-generation laser for high-resolution spectroscopy in the $667-865 \mathrm{~cm}^{-1}$ range," Proc. SPIE. 11670, Nonlinear Frequency Generation and Conversion: Materials and Devices XX, 116700A (2021).

[16] Sharpe, S. W., Johnson, T. J., Sams, R. L., Chu, P. M., Rhoderick, G. C. and Johnson, P. A., "Gas-phase databases for quantitative infrared spectroscopy," Appl. Spectrosc. 58(12), 1452-1461 (2004).

[17] Hollenstein, H., Piccirillo, S., Quack, M. and Snels, M., "High-resolution infrared spectrum and analysis of the $\mathrm{v}_{11}, \mathrm{~A}_{2 \mathrm{u}}\left(\mathrm{B}_{2}\right)$ fundamental band of ${ }^{12} \mathrm{C}_{6} \mathrm{H}_{6}$ and ${ }^{13} \mathrm{C}^{12} \mathrm{C}_{5} \mathrm{H}_{6}$," Mol. Phys. 71(4), 759-768 (1990).

[18] Gordon, I. E., Rothman, L. S., Hill, C., Kochanov, R. V., et al., "The HITRAN2016 molecular spectroscopic database," J. Quant. Spectrosc. Radiat. Transf. 203, 3-69 (2017). 